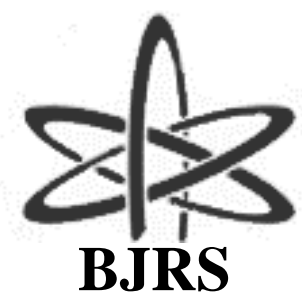

\author{
BRAZILIAN JOURNAL \\ $\mathrm{OF}$ \\ RADIATION SCIENCES \\ 06-02-B (2018) 01-12
}

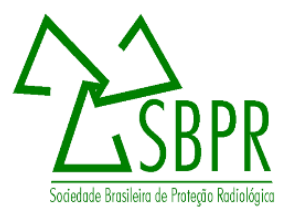

\title{
Overview of Brazilian industrial radiography accidents with cutaneous radiation syndrome
}

\author{
Lima $^{a}$ C. M. A.; Da Silva ${ }^{\text {b F. C. A. }}$ \\ ${ }^{a}$ Postgraduate Course Stricto Sensu in Radiation Protection and Dosimetry, Institute of Radiation Protection and \\ Dosimetry/CNEN, 22783-127, Rio de Janeiro-RJ, Brasil \\ ${ }^{b}$ Institute of Radiation Protection and Dosimetry/CNEN, 22783-127, Rio de Janeiro-RJ, Brasil \\ dasilva@ird.gov.br
}

\begin{abstract}
Many of industrial radiological accidents are related to industrial gamma radiography, which makes this practice to have a high potential risk for accidental exposures to ionizing radiation. More than 80 radiological accidents occurred in the world that includes 6 Brazilian accidents with Cutaneous Radiation Syndrome (terminology used in Brazil and Latin America) or Local Radiation Injury (terminology is used in Europe, USA and by IAEA). From 1985 to 2000 five Brazilian accidents happened with ${ }^{192} \mathrm{Ir}$ and one with ${ }^{60} \mathrm{Co}$ radioactives sources. Nineteen members of the public and 8 radiographers were involved. All of them suffered severe hands and fingers injuries. Brazilian accidents were analyzed showing the main causes, the consequences for workers, estimated radiation doses and main lessons learned. This paper describes a detailed summary of each radiological accident in industrial gamma radiography occurred in Brazil.
\end{abstract}

Keywords: Industrial radiography, radiological accidents, Cutaneous Radiation Syndrome.

ISSN: 2319-0612

Accepted 2018-07-31 


\section{INTRODUCTION}

Industrial radiography is one of the non-destructive inspection methods widely used in the evaluation of ferrous and non-ferrous materials welds, castings and forgings, where the quality requirements of the industrial sector are necessary to avoid discontinuities in parts, components, equipment, etc. This method uses radioactive sources of ${ }^{60} \mathrm{Co},{ }^{75} \mathrm{Se}$ and especially ${ }^{192} \mathrm{Ir}$ [1]. Industrial radiography is classified as Category 2 in the IAEA Categorization System [2] because the sources have high activity and consequently greater radiological risk.

Industrial radiography work is often carried out under difficult working conditions, such as inside confined spaces, in extreme cold or hot temperature, or during the night. Working under such adverse conditions might result in operational situations in which occupational radiation protection procedures may be compromised. Experience shows that incidents involving industrial radiography sources have sometimes resulted in high doses to workers and even to members of the public, causing severe health consequences such as Cutaneous Radiation Syndrome (CRS) and, in a few cases, death [3].

In the last 30 years, more than 80 severe radiological accidents in industrial radiography involving 120 radiation workers, 110 members of the public and 12 deaths happened in the world, including 10 accidents in Latin America [4, 5, 6, 7]. IAEA published three Latin America reports: Peru (1999), Bolivia (2002) and Chile (2005). In these events only members of the public were involved. A brief overview of these 3 events is presented below:

1. PERU (1999): A welder picked up a ${ }^{192}$ Ir radioactive source, with $1.37 \mathrm{TBq}$ of activity, used in industrial gamma radiography and left it in his pants pocket for several hours. He received a high localized radiation dose and had his leg amputated. Ultimately he had a hip desarticulation. His wife and children were also exposed to low doses. Main lesson learned: Persons not directly working with radiation sources, but working nearby, should receive appropriate information and training [8].

2. BOLIVIA (2002): An accident involving an industrial radiography source containing $0.67 \mathrm{TBq}$ of ${ }^{192}$ Ir occurred in Cochabamba, Bolivia, some $400 \mathrm{~km}$ from the capital, La Paz. A faulty radiography source container had been sent back to the headquarters of the company concerned 
in La Paz together with other equipment as cargo on a passenger bus. This gave rise to a potential for serious exposure to the bus passengers as well as the company employees who were using_and transporting the source. Main lesson learned: Employers should provide suitable and adequate human resources and appropriate training in radiation protection and safety and periodic retraining can help to ensure that the required degree of competence is maintained [9].

3. CHILE (2005): During a cellulose plant construction a ${ }^{192} \mathrm{Ir}$ radioactive source, with $3.33 \mathrm{TBq}$ of activity, fell out of industrial gamma radiography equipment unnoticed, and was found later and handled by three scaffolding workers. Main lesson learned: Safe operation of industrial gamma radiography depends crucially on proper implementation of radiation protection and safety, for example, the regular use of alarm monitors and dose rate meters [10].

Brazilian data includes 6 serious radiological accidents affecting 8 radiation workers and 19 members of the public, resulting in the development of Cutaneous Radiation Syndrome (also called by some as "local radiation injury" or improperly "radiation burns") in hands and fingers [6, 7]. This paper describes each radiological accident in industrial gamma radiography occurred in Brazil.

\section{MATERIALS AND METHODS}

A detailed analysis of 6 Brazilian radiological accidents with Cutaneous Radiation Syndrome in industrial radiography was performed based on published papers, thesis and reports. The analysis was made taking into account the following aspects of the accident: main causes, consequences for workers, estimated radiation doses and main lessons learned.

\section{RESULTS AND DISCUSSION}

Five accidents occurred with ${ }^{192}$ Ir radioactive source used in mobile industrial gamma devices (1985 (2 events), 1988, 1997, 1998) and one with ${ }^{60}$ Co radioactive source in fixed device (2000). Nineteen members of the public were involved in two events (1985) and eight radiation workers in four events (1988, 1997, 1998 and 2000). All of them received high radiation doses and suffered Cutaneous Radiation Syndrome in hands and fingers. 


\subsection{Brazil (1985 - $1^{\text {st }}$ event) [6]:}

\section{a) Event:}

An industrial gamma radiography team worked using a gamma device (Gammamat TIF) with the broken key inside the lock. In the morning, after a night of work, the team returned to the company's headquarter to store the gamma device inside the bunker. A radiation worker removed the device from transport box to the safety bunker and left the box at the company parking. But he did not realize that the ${ }^{192}$ Ir radioactive source with $3.28 \mathrm{TBq}$ of activity had fallen inside the box. A security person found the radioactive source and handled it for 30 seconds. After seeing a skull painted in the pigtail, he left the source inside the box. The radioactive source stayed at the company parking during 10 hours without shielding. All company workers and children in a neighbour primary school were irradiated during this time.

b) Main causes:

- The gamma radiography device was not checked before working and it was used with the broken key inside the lock.

- The industrial radiography company had not maintenance program for of gamma radiography devices.

- Radiation protection and safety procedures were not followed.

c) Main consequences:

The security person had Cutaneous Radiation Syndrome on the left and right hands, including blisters, necrosis and atrophy of the fingers of the left hand and the right index finger.

d) Radiation doses:

- Effective dose: no personal dosimeter was used.

- Cytogenetic estimation: it was not done.

- Equivalent dose estimated by mathematics calculations:

- $\quad$ hands: $410.54 \mathrm{~Gy}$; - eye lens: $0.26 \mathrm{~Gy}$; - gonads: 0.04Gy

e) Main lessons learned:

- Safety and protection checks must be done before using the gamma device.

- The use of any gamma device must be prohibited with any problem, especially with the broken key inside the lock. 
- A special transport box must be used during the transport.

- A fixed radiation alarm monitor must be installed in the parking area.

\subsection{Brazil (1985 - 2nd event) [6]:}

a) Event:

An industrial gamma radiography device (Gammamat TIF), with a ${ }^{192}$ Ir radioactive source with $0.88 \mathrm{TBq}$ of activity, was stolen from the transport vehicle. A truck driver found a box at the road and took it to his company. At the company the truck driver opened the box and found the gamma radiography device with the key. He showed the device to his friends and opened it. The radioactive source was removed and handled by all. At the end he put back the source inside the device. Ten persons were overexposure and 6 handled the source. The truck driver took the device to his home and showed to 6 people: wife, daughter and 4 friends. The gamma radiography device was kept in the driver truck house for 10 days.

b) Main causes:

- The key was left attached in the gamma device.

- The transport vehicle was left unattended during the lunch time.

- Radiation protection and safety procedures were not followed.

c) Main consequences:

The members of public (MP) including the driver truck had Cutaneous Radiation Syndrome on the hands, mainly in the fingers.

- MP1: injuries on the right index finger and left thumb.

- MP2: injuries on the left middle and thumb fingers.

- MP6: injuries, loss of movement and atrophy on the right index, middle and thumb fingers (driver tuck).

- MP7: injuries on the right index and thumb fingers.

- MP10: injuries on the right index and thumb fingers.

- MP11: injuries on the right hand and little finger.

- MP18: injuries on the left thumb, index and middle fingers.

d) Radiation doses:

- Effective dose: no personal dosimeter was used. 
- Cytogenetic estimation: it was not done.

- Equivalent dose in the hands estimated by mathematics calculations:

- $\quad$ MP1: $25.00 \mathrm{~Gy}$;

- $\quad$ MP2: $18.72 \mathrm{~Gy}$;

- $\quad$ MP6: 162.24 Gy (driver tuck);

- $\quad$ MP7: 21.84 Gy;

- Equivalent dose in the eye lens estimated by mathematics calculations:

- $\quad$ MP1: $2.73 \mathrm{mGy}$;

- $\quad$ MP2: $2.04 \mathrm{mGy}$;

- $\quad$ MP6: $17.72 \mathrm{mGy}$ (driver tuck);

- $\quad$ MP7: 2.39 mGy;

- Equivalent dose in the gonads estimated by mathematics calculations

- $\quad$ MP1: $2.54 \mathrm{mGy}$

- $\quad$ MP2: $2.91 \mathrm{mGy}$;

- $\quad$ MP6: $10.90 \mathrm{mGy}$ (driver tuck);

- $\quad$ MP7: 1,72 mGy;

e) Main lessons learned:

- The transport vehicle must be permanently attended during all time.

- The transport box must be fixed in the vehicle to be safer.

- The gamma device key must be kept with the radiation worker and never be left in the lock during transport.

\subsection{Brazil (1988) [6]:}

a) Event:

During an industrial gamma radiography operation, with a ${ }^{192} \mathrm{Ir}$ radioactive source with 3.29 $\mathrm{TBq}$ of activity, the source holder disconnected of the control cable and kept inside the guide tube. The radiation workers handled the guide tube, with the radiation source inside it, three times to prepare more assays.

b) Main causes: 
- Radiation workers did not use the radiation monitor during radiography assay.

- The source holder was not properly connected to the control cable.

c) Main consequences:

Three radiation workers (W) had Cutaneous Radiation Syndrome on the hands mainly in the fingers.

- W1: injuries in thumb, index and middle fingers.

- W2: injuries in index and middle fingers.

- W3: injuries in index and middle fingers of both hands.

d) Radiation doses:

- Effective dose using film badge dosimeter:

- $\quad$ W1: $300.42 \mathrm{mSv}$; - W2: $264.32 \mathrm{mSv}$; - W3: $304.44 \mathrm{mSv}$;

- Cytogenetic estimation: it was not done.

- Equivalent dose in hands estimated by mathematics calculations:

W1: 74.25 Gy; - W2: 30.95 Gy; - W3: 33.13 Gy (left hand) and 55.69 Gy (right hand);

- Equivalent dose in eye lens estimated by mathematics calculations

- $\quad$ W1: 0.18 mGy; - W2: 0.26 mGy; - W3: 0.24 mGy;

- Equivalent dose in gonads estimated by mathematics calculations

- $\quad$ W1: 0.10 mGy; - W2: 0.12 mGy; - W3: 0.14 mGy;

e) Main lessons learned:

- To use continually the radiation monitor during radiography operation.

- To check the right connection between the source holder and the control cable.

\subsection{Brazil (1997) [7]:}

a) Event:

After four industrial gamma radiography exposures, using a gamma device Iriditron 520, with a

${ }^{192} \mathrm{Ir}$ radioactive source with $1.62 \mathrm{TBq}$ of activity, a radiation worker (trainee operator) was designated to prepare another series of exposures. He prepared the arrangement and made himself four more exposures. When the Responsible for Open Installation (ROI) entered to prepare another series, he observed that his personal alarm monitor was indicating a high level radiation inside the 
bunker. He realized that the radioactive source was outside the shielding. The Responsible for Open Installation (ROI) planned and carried out the source rescue with the same team. It was observed that the source was disconnected from its control cable and remained at the end of the guide tube near the collimator. After several attempts, the trainee operator took off the collimator. As the source holder was fixed on the collimator, he held the source holder with his hands and put it inside the guide tube. Using a drive cable they pushed the source to the shielded exposure device.

b) Main causes:

- The team was not complete because an authorized operator was missing.

- The radiography assays were performed only by the trainee operator.

- The team performed all radiography exposures without a survey meter that was left outside of the bunker.

- The trainee operator did not use the personal alarm monitor.

- The trainee operator did not connect correctly the source holder to the control cable.

- The responsible planned and carried out the source rescue with the same team.

- The emergency rescue was performed by the trainee operator. He was the team member with the highest dose.

- A wrong emergency rescue procedure was applied because the trainee operator held the source holder with his own hand.

c) Main consequences:

The radiation worker (trainee operator) had injuries on the index finger of the left hand with erythema and blister.

d) Radiation doses:

- Effective dose: $345.50 \mathrm{mSv}$, using thermoluminescent dosimeter.

- Cytogenetic estimation: $180.00 \mathrm{mGy}$.

- Equivalent dose in fingers of left hand: 8.25 Gy, by mathematics calculations.

e) Main lessons learned:

- All workers must use personal alarm monitor during their job.

- Refresh training must be done annually with emphasis in emergency and accident situations.

- Continuous training about gamma radiography operations must be done for all workers. 


\subsection{Brazil (1998) [7]:}

\section{a) Event:}

Just after the first exposure, inside a bunker, using an industrial gamma radiography device Gamma Century with a ${ }^{192}$ Ir radioactive source with $0.95 \mathrm{TBq}$ of activity, the radiation worker did not return the source to the device. As he was working alone, he prepared the setup to another exposure without checking the radiation with an area monitor. During the preparations he handled the guide tube with the source inside it. He worked with the source exposed for more five assays. At the end he used the area and alarm monitors that were near the bunker door and realized the high radiation inside the bunker. The worker's hands were in contact with the radioactive source six times.

b) Main causes:

- The gamma radiography assays were performed by only one radiation worker.

- Radiation areas and alarm monitors were not used by the radiation worker.

- The radiation worker had not knowledge in how to work with the gamma device.

- The gamma device was not locked after each exposure ending.

- General and specific training of protection and safety procedures were not provided.

c) Main consequences:

The radiation worker had injuries on the right thumb with erythema and blister.

d) Radiation doses:

- Effective dose: $300.00 \mathrm{mSv}$, using thermoluminescent dosimeter.

- Cytogenetic estimation: $180.00 \mathrm{mGy}$

- Equivalent dose in hands: it was not estimated.

e) Main lessons learned:

- All workers must use personal alarm monitor during their work.

- Initial and annual refresh training about gamma radiography operations must be done for all workers.

- Audit program to check the workers performance in radiation protection must be performed by the Company. 


\subsection{Brazil (2000) $[11,12,13]$ :}

a) Event:

An industrial gamma radiography operator was performing routine exposures with a ${ }^{60} \mathrm{Co}$ Gammamat device (TK100, Isotopen-Technik) containing a radioactive source with $2.11 \mathrm{TBq}$ of activity. The operator was not bearing a personal alarm monitor, and did not carry a survey meter. At the end of the tasks, when he tried to detach the guide tube from the gamma device - unaware that the source was not in the safe position - he kept his left hand very close to the radioactive source, for approximately 30 seconds. Noticing that it was impossible to detach the guide tube from the exposure container area monitoring was conducted, which showed that indeed the source was not in the secured position.

b) Main causes:

- The radioactive source was stuck inside the exposure container, in unsafe position, probably due to corrosion inside the container and poor maintenance of the equipment.

- The operator was not using any personal alarm monitor.

- The operator did not perform radiation monitoring of the gamma device after the assay.

- The operator did not follow the radiation and safety protection procedures.

c) Main consequences:

The industrial gamma radiography operator had Cutaneous Radiation Syndrome on the fingers of the left hand, including the appearance of erythema, oedema and blisters.

d) Radiation doses:

- Effective dose: $88.10 \mathrm{mSv}$ using film badge dosimeter.

- Cytogenetic estimation: lower than $60.00 \mathrm{mGy}$.

- Equivalent dose in the hands estimated by "Visual Monte Carlo Dose Calculation Brazilian Software": left thumb: 7.80 Gy and left index finger: $15.90 \mathrm{~Gy}$;

e) Main lessons learned:

- The internal gamma device channel must be periodically inspected using an industrial borescope and be smeared to check any contamination.

- Audible and vibrating alarm monitors must be used by all radiography operators. 
- Education and training in radiation protection and safety must be continuous provided to operators.

\section{CONCLUSION}

The review of these radiological accidents clearly demonstrates that industrial gamma radiography has a high potential risk for overexposures.

The main consequence of these radiological accidents for radiation workers and, unfortunately, for members of the public can be the Cutaneous Radiation Syndrome, such as, blisters, erythema, oedema, necrosis, atrophy until amputation, generally in hands and fingers.

Radiological accidents in industrial gamma radiography could be avoided if the company director and the radiation protection officer provide, at least, the following aspects: continuous education and training in radiation protection, safety and security; individual monitor with audible and vibrating alarms to radiation workers; implementation of audit program to verify the workers performance during an industrial radiography assay.

Brazilian radiation doses in hands, eye lens and gonads were estimated using mathematics calculations, due to the absence of updated methodology. So a review of these doses using, for example, Monte Carlo method is strongly recommended.

\section{ACKNOWLEDGMENT}

The authors are grateful to $\mathrm{CNPq} / \mathrm{Brazil}$ to grant a M.Sc. Scholarship that made this work possible.

\section{REFERENCES}

[1] LIMA, C. M. A. Análise de acidentes e incidentes radiológicos em radiografia industrial. Monografia Pós-Graduação IRD, Rio de Janeiro, 2011. 
[2] IAEA - International Atomic Energy Agency. Categorization of Radioactive Sources. Safety Standards Series RS-G-1.9, Vienna, 2005.

[3] IAEA - International Atomic Energy Agency. The information System on Occupational Exposure in Medicine, Industry and Research (ISEMIR): Industrial Radiography. TECDOC 1747, Vienna, 2014.

[4] IAEA - International Atomic Energy Agency. Lessons learned from accidents in industrial radiography. Safety Reports Series 7. Vienna, 1998.

[5] UNSCEAR - United Nations Scientific Committee on the Effects of Atomic Radiation. Sources and effects of ionizing radiation - radiation exposures in accidents - Annex C. UNSCEAR Report, Vienna, 2011.

[6] DA SILVA, F. C. A. Uma metodologia de análise de acidentes radiológicos em gamagrafia industrial. Tese Mestrado IME, Rio de Janeiro, 1990.

[7] DA SILVA, F.C.A., HUNT, J.G., RAMALHO, A.T, PINTO L. Reconstructive dosimetry - A study of a radiological accident in Brazilian industrial gammagraphy. Proceedings of the International Conference on Occupational Monitoring, Geneva (2002).

[8] IAEA - International Atomic Energy Agency. The radiological accident in Yanango, IAEA, 2000.

[9] IAEA - International Atomic Energy Agency. The radiological accident in Cochabamba, IAEA, 2004.

[10] IAEA - International Atomic Energy Agency. The radiological accident in Nueva Aldea, IAEA, 2009.

[11] DA SILVA, F. C. A. Dosimetria reconstrutiva de acidentes radiológicos usando o método de Monte Carlo. Tese Doutorado COPPE/UFRJ, Rio de Janeiro, 2003.

[12] DA SILVA, F. C. A.; HUNT, J. G.; RAMALHO, A. T.; CRISPIM, V. R. Dose reconstruction of a Brazilian industrial gamma radiography partial-body overexposure case. J Radiol Prot 25: 289-298, 2005. doi: 10.1088/0952-4746/25/3/005.

[13] C. M. A. LIMA, A. R. LIMA, A. L. DEGENHARDT, N. J. VAlVERDE, F. C. A. DA SILVA. Reconstructive dosimetry for cutaneous radiation syndrome. Braz J Med Biol Res 48(10): 895-901, 2015. doi: 10.1590/1414-431X20144337. 